\title{
Analytical Modelling of Perforated Geometrical Domains by the R-functions
}

\section{Yuriy Semerich}

\author{
Limited Liability Company Budgeting and Financial Technologies \\ Pushkina str. 2, 440008, Penza, Russia \\ E-mail(corresp.): y.semerich@bftcom.com \\ E-mail: yu.semerich@gmail.com
}

Received September 13, 2019; revised May 30, 2020; accepted June 1, 2020

\begin{abstract}
This paper deals with the construction of boundary equations for geometric domains with perforation. Different types of perforated geometric domains are considered. The R-functions method for analytical modelling of perforated geometrical domains is used. For all constructed equations, function plots are obtained.
\end{abstract} Keywords: perforated domain, R-functions, R-operations, boundary equation of domain.

AMS Subject Classification: 14H50; 26B40; 51M15; 65D17.

\section{Introduction}

The beauty of the whole world outside created by a human being is striking in its diversity. However, a detailed examination of all this diversity shows many similarities. For example, what could be common between window holes in buildings and road markings at pedestrian crossings or a washing machine drum and a colander? The answer - it is symmetry! The number of definitions for the term symmetry is almost the same amount as the types of symmetry. This topic is described in more detail in the last work of the outstanding German mathematician Hermann Weyl [9].

In this paper, attention will be paid to one type of symmetry which is called translational symmetry and occurs in the perforation. In general, perforation can be understood as a significant number of correctly placed holes of regular shape, obtained in the process of perforation in any material. Perforation is used in many fields of human activity. For example, it is used in beams and it allows to make lighter building constructions. In such areas of industry as the automotive industry, shipbuilding, and aircraft, perforation allows improving 
the technical characteristics of the manufactured samples. In the food industry and agriculture, perforation is used in the details of mechanisms and machines for cleaning and separation processes. Perforation is often used as a design element in an urban environment. But the most important thing is that perforation is the subject of research in scientific practice. Problems of various fields calculating perforated domains are very important $[1,2,3]$. To solve such problems, the class of grid methods is often used. Another class of methods for solving applied problems is meshless methods. One of these methods is the R-function method [4]. Its main feature is the construction of boundary equations in an analytical form for geometric domains of arbitrarily complex form without approximation.

\section{System of the R-functions and properties of the boundary domain equation}

In the R-functions method, the boundary equation of domain $\Omega$ is given implicitly

$$
\omega(x, y)=0,
$$

where the function $\omega(x, y)$ satisfies the following conditions:

$\omega(x, y)>0, \forall(x, y) \in \Omega, \omega(x, y)=0, \forall(x, y) \in \partial \Omega, \omega(x, y)<0, \forall(x, y) \notin \Omega$.

Additionally, the condition of normalization is formulated as

$$
\left.\frac{\partial \omega}{\partial \vec{n}}\right|_{\partial \Omega}=1,\left.\frac{\partial^{k} \omega}{\partial \vec{n}^{k}}\right|_{\partial \Omega}=0,(k=2,3, \ldots, m),
$$

where $\vec{n}$ is the internal normal vector to boundary $\partial \Omega$, defined at its regular points. The construction of a function $\omega(x, y)$ with the specified properties is performed using the following R-functions:

$$
\begin{gathered}
x \wedge_{\alpha} y \equiv \frac{1}{1+\alpha}\left(x+y-\sqrt{x^{2}+y^{2}-2 \alpha x y}\right), \\
x \vee_{\alpha} y \equiv \frac{1}{1+\alpha}\left(x+y+\sqrt{x^{2}+y^{2}-2 \alpha x y}\right), \quad \bar{x} \equiv-x .
\end{gathered}
$$

Here $x \wedge_{\alpha} y$ is called $R$-conjunction, $x \vee_{\alpha} y$ is called $R$-disjunction, $\bar{x}$ is called $R$-negation. The parameter $\alpha(x, y)$ has the property $\alpha(x, y) \equiv \alpha(y, x) \equiv$ $\alpha(-x, y) \equiv \alpha(x,-y)$ and satisfies the inequalities $-1<\alpha(x, y) \leq 1$. For example, if $\alpha(x, y)=1$ then it has a simpler form

$$
x \wedge_{1} y \equiv \min (x, y), \quad x \vee_{1} y \equiv \max (x, y) .
$$

Moreover, $\min (x, y)+\max (x, y) \equiv x+y, \min (x, y) \max (x, y) \equiv x y$. In this connection, $\min (x, y)$ and $\max (x, y)$ can be considered as the smaller and the larger root of the equation $z^{2}-(x+y) z+x y=0$, i.e.

$$
\min (x, y) \equiv \frac{1}{2}(x+y-|x-y|), \quad \max (x, y) \equiv \frac{1}{2}(x+y+|x-y|) .
$$

For convenience, we will use $\alpha(x, y)=0$ in the rest. The mathematical framework of the $\mathrm{R}$-functions theory is described in $[4,5]$. 


\section{Definitions and a problem statement}

Let us use the R-functions theory for construction boundary equations of perforated geometric domains. Consider an unbounded open multiply-connected domain $\Omega$ on the plane $O x y$ and its subdomain $\Omega_{0}, \Omega_{0} \subset \Omega \subset \mathbb{R}^{2}$.

Definition 1. Subdomain $\Omega_{0}$ is called a perforation grid and defined by a function $\omega_{0}(x, y)$.

Definition 2. Domain $\Omega$ is called a perforated domain if a function $\omega(x, y)$ satisfies condition

$$
\omega(x, y)=\omega_{0}\left(x \pm q h_{x}, y \pm q h_{y}\right)
$$

where $q \in \mathbb{Z}$ and $h_{x}, h_{y}$ are vector components of $\vec{h}=\left(h_{x}, h_{y}\right)$.

Let us regard $\Omega_{0}$ as five-connected and shown in Figure 1.

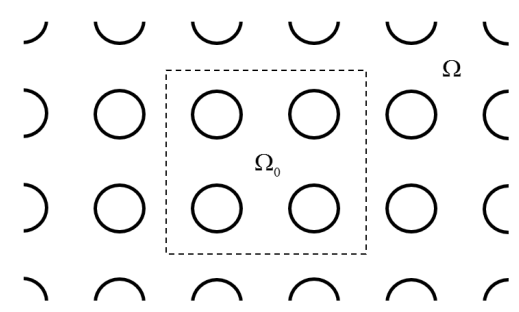

Figure 1. Perforated domain $\Omega$ and its subdomain $\Omega_{0}$.

Definition 3. The holes of the perforation grid $\Omega_{0}$ are called its elements.

Let us determine the interrelation between elements of the perforation grid $\Omega_{0}$ as shown in Figure 2. Here $d_{12}$ is the distance between the centers of the

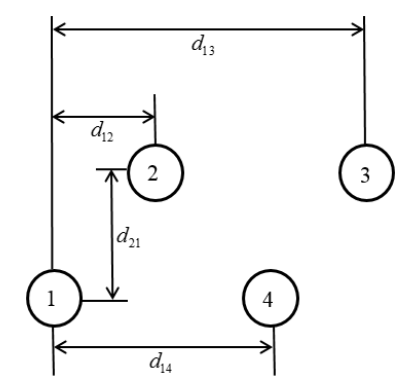

Figure 2. The interrelation between elements of the perforation grid $\Omega_{0}$.

elements " 1 " and "2" in the projection on the $x$-axis; $d_{21}$ is the distance between the centers of the elements " 1 " and "2" in the projection on the $y$-axis; $d_{14}$ is the distance between the centers of the elements "1" and "4" in the projection on the $x$-axis; $d_{13}$ is the distance between the centers of the elements " 1 " and " 3 " in the projection on the $x$-axis. 
For definiteness, let us set the center of the element "1" at the beginning of the Cartesian coordinate system $O x y$ and will assume that it can be separated from the other elements using stripes along the $x$-axis and the $y$-axis of the same width $2 a$, that is, from $-a$ to $a$ as shown in Figure 3.

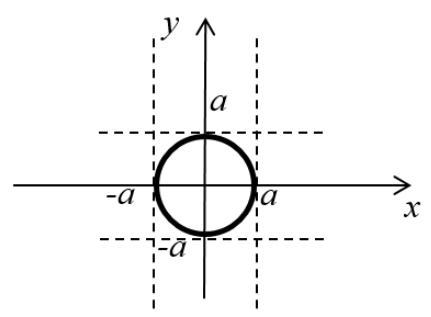

Figure 3. Element "1" of the perforation grid $\Omega_{0}$.

On the basis of the considerations outlined above, the problem statement of boundary equation building for perforated domain is formulated as - construct a function $\omega(x, y)=0$ with the described properties and satisfying Definition 2 . For these purposes, we use the apparatus of the R-functions theory. However, the presence of a large number of holes in the perforated region will lead to inconveniences associated with a large number of R-operations and functions involved in the construction of a $\omega(x, y)=0$. It is important to emphasize that such a problem was not previously considered by any author, therefore, it is formulated and solved for the first time. However, as will be shown later, to solve this problem, it will be necessary to use the method of geometric symmetries taking into account.

\section{Boundary equations of perforated geometric domains}

Note that the problem of boundary equations construction for the perforated geometric domains can be considered as a generalization of the problem of boundary equations construction for the domain with a translational-type symmetry. For example, the translational domain can be considered as a single-row perforated domain. Due to this connection, we will use the methods described in the work $[6,7,8]$.

\subsection{Perforated domain with densely filled elements of perforation grid}

Let us consider the simplest case of a perforated domain $\Omega$. For this case we will use a unit radius circle, i.e. $a=1$, as the element " 1 " of the perforation grid $\Omega_{0}: \omega_{1}(x, y) \equiv 1-x^{2}-y^{2}$. Such function is even on $x$ and $y$, i.e. $\omega_{1}(-x,-y)=\omega_{1}(x, y)$.

The interrelation distances of the perforation grid $\Omega_{0}$ are these: $d_{12}=0$, $d_{14}=d_{21}=d_{13} \cong 2 a$. We obtain the domain $\Omega$ shown in Figure 4 .

In Figure 4, the elements of the perforation grid $\Omega_{0}$ are highlighted and form vertices of a square. 


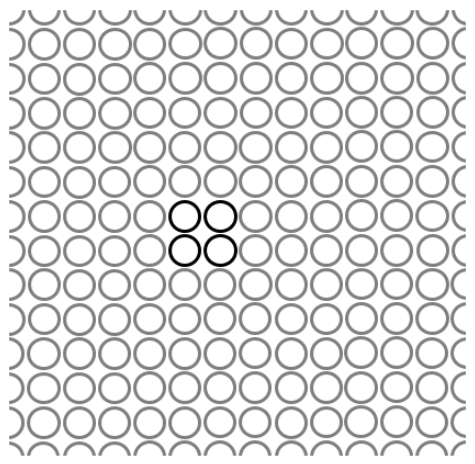

Figure 4. Domain $\Omega$.

Let us consider the function

$$
\mu(x, h)=\frac{4 h}{\pi^{2}} \sum_{i=1}^{n} \frac{(-1)^{i+1}}{(2 i-1)^{2}} \sin \left[\frac{(2 i-1) x \pi}{h}\right]
$$

that is a Fourier series expansion of a sawtooth function along the $x$-axis [8]. Here $h$ is a half period. Then set the boundary equation for the domain $\Omega$ as follows

$$
\omega_{d_{14}, d_{21}}(x, y) \equiv-\omega_{1}\left(\mu\left(x, d_{14}\right), \mu\left(y, d_{21}\right)\right)=0 .
$$

Plots of the function $\omega_{d_{14}, d_{21}}(x, y)$ shown in Figure 5 for level lines and in perspective view.
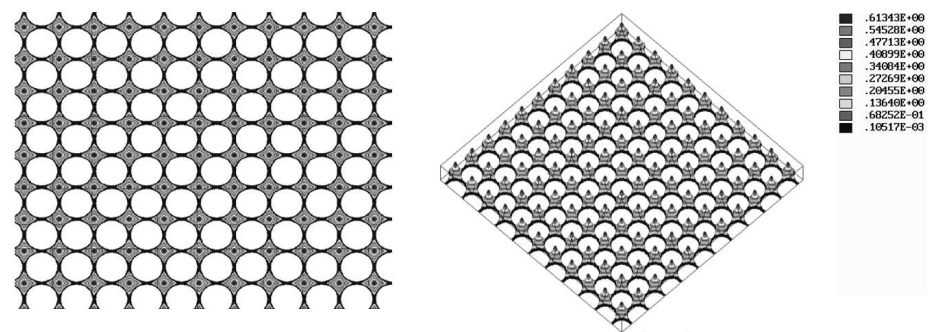

Figure 5. Plots of the function $\omega_{d_{14}, d_{21}}(x, y)$ for subsection 4.1.

\subsection{Perforated domain with equidistant elements of perforation grid}

For the case $d_{14}=d_{21}=4 a\left(d_{12}=0, d_{13}=4 a\right)$ in Equation (4.1), then it result for a perforated domain with elements of the perforation grid $\Omega_{0}$ located at the same distance from each other (Figure 6).

\subsection{Perforated domain with elements of perforation grid forms the vertices of a rectangle with a length along the $x$-axis}

Let us consider a case for the elements of the perforation grid $\Omega_{0}$ forms the vertices of a rectangle. Note that for such case it would be enough to change 

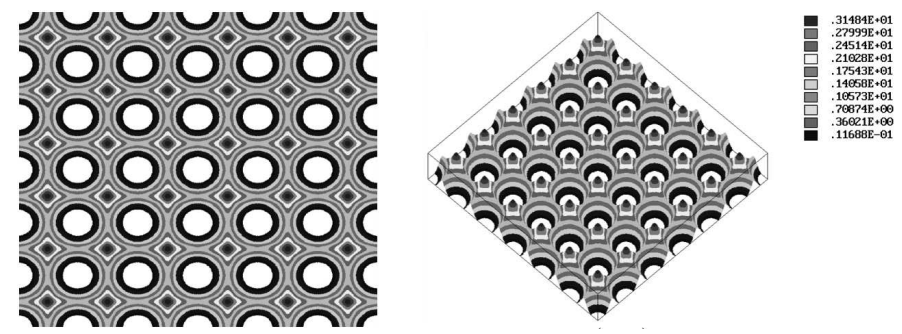

Figure 6. Plots of the function $\omega_{d_{14}, d_{21}}(x, y)$ for subsection 4.2.

the values $d_{14}\left(d_{13}\right)$ from the previous case. However, we proceed another way and construct an equation that will allow us to take into account other cases in which the elements of the perforation grid $\Omega_{0}$ form the vertices of more complex shapes.

Set a values of parameters as $d_{12}=0,2 d_{14}=3 d_{21}=12 a,\left(d_{13}=6 a\right)$. In this case, the elements of the perforated grid $\Omega_{0}$ define vertices of a rectangle with a length along the $x$-axis. Then a boundary equation of the domain $\Omega$ can be written as

$$
\begin{gathered}
\omega_{d_{12}, d_{14}, d_{21}}(x, y) \equiv-\left[\left\{\omega_{1}\left(\mu\left(x, d_{14}\right), \mu_{2}\left(y, d_{21}\right)\right) \wedge_{0} \cos \left(\mu\left(y, d_{21}\right)\right)\right\}\right. \\
\vee_{0}\left\{\omega_{1}\left(\mu\left(x-d_{12}, d_{14}\right), \mu\left(y-d_{21}, d_{21}\right)\right)\right. \\
\left.\left.\wedge_{0} \cos \left(\mu\left(y-d_{21}, d_{21}\right)\right)\right\}\right]=0 .
\end{gathered}
$$

Figure 7 shows plots of the function $\omega_{d_{12}, d_{14}, d_{21}}(x, y)$ from (4.2) as a picture of the level lines and in perspective view.
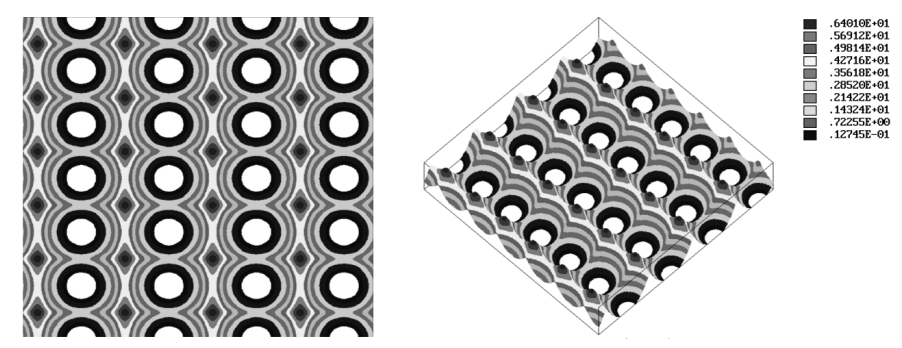

Figure 7. Plots of the function $\omega_{d_{12}, d_{14}, d_{21}}(x, y)$ for subsection 4.3.

\subsection{Perforated domain with elements of perforation grid forms the vertices of a rectangle with a length along the $y$-axis}

To construct the boundary domain equation for a case the elements of the perforated grid $\Omega_{0}$ define vertices of a rectangle with a length along the $y$-axis, we use the next values of parameters in the Equation (4.2): $d_{12}=0,3 d_{14}=$ $2 d_{21}=12 a,\left(d_{13}=4 a\right)$. As a result of such assumptions, plots of the function $\omega_{d_{12}, d_{14}, d_{21}}(x, y)$ as a picture of the level lines and in perspective view is shown in Figure 8. 

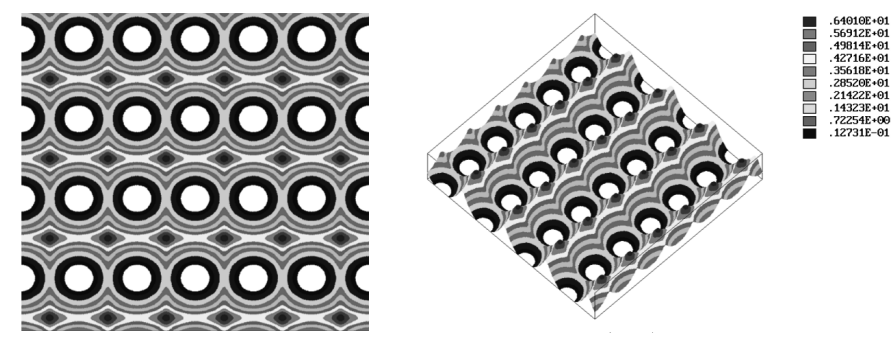

Figure 8. Plots of the function $\omega_{d_{12}, d_{14}, d_{21}}(x, y)$ for subsection 4.4.

\subsection{Perforated domain with elements of perforation grid forms the vertices of an equilateral parallelogram}

Let us complicate the shape of the perforation grid $\Omega_{0}$ and consider a case the elements forms the vertices of an equilateral parallelogram. To construct the boundary equation for a such case, the Equation (4.2) with the next values of the numerical parameters: $2 d_{12}=d_{14}=d_{21}=4 a,\left(d_{13}=4 a\right)$ is using. In this case, plots of the function $\omega_{d_{12}, d_{14}, d_{21}}(x, y)$ as a picture of the level lines and in perspective view is shown in Figure 9.
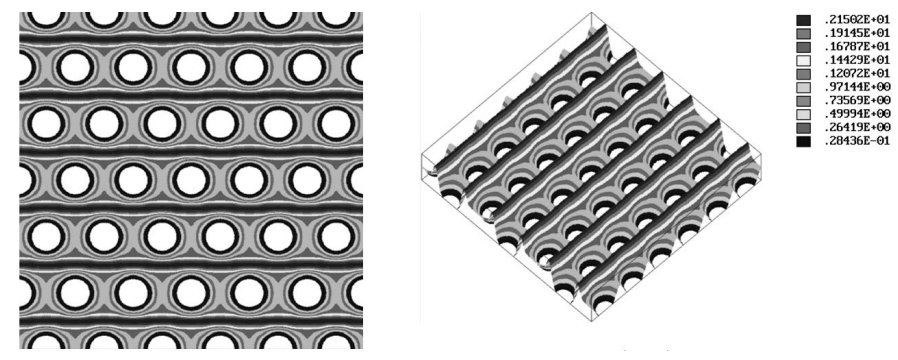

Figure 9. Plots of the function $\omega_{d_{12}, d_{14}, d_{21}}(x, y)$ for subsection 4.5.

4.6 Perforated domain with elements of perforation grid forms the vertices of a parallelogram with a longer length along the $\boldsymbol{x}$-axis

Just as it was done for a rectangle, consider a case when the elements of the perforation grid $\Omega_{0}$ form the vertices of a parallelogram with a longer length along the $x$-axis. To realize this case, it is necessary to change values of the numerical parameters at the Equation (4.2) according to next: $6 d_{12}=2 d_{14}=3 d_{21}=12 a$ $\left(d_{13}=6 a\right)$. At Figure 10 shows plots of the function $\omega_{d_{12}, d_{14}, d_{21}}(x, y)$ as a picture of the level lines and in perspective view.

4.7 Perforated domain with elements of perforation grid forms the vertices of a parallelogram with a longer length along the $y$-axis

If the elements of the perforation grid $\Omega_{0}$ forms the vertices of a parallelogram with a longer length along the $y$-axis, it is necessary to set values of numerical 

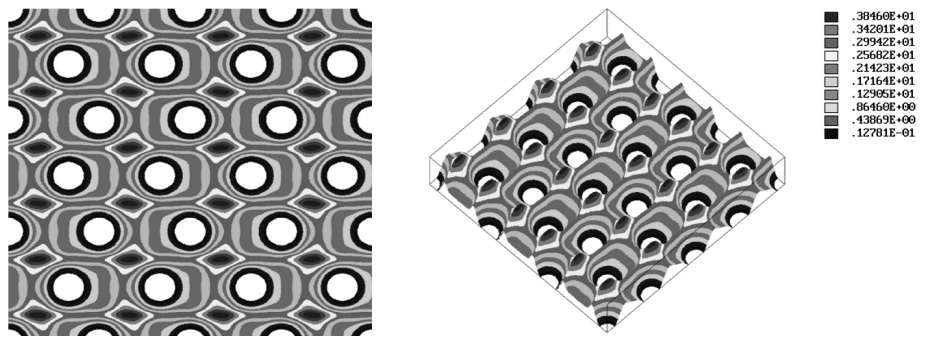

Figure 10. Plots of the function $\omega_{d_{12}, d_{14}, d_{21}}(x, y)$ for subsection 4.6.

parameters according to next: $6 d_{12}=3 d_{14}=4 d_{21}=12 a\left(d_{13}=4 a\right)$. Then plots of the function $\omega_{d_{12}, d_{14}, d_{21}}(x, y)$ as a picture of the level lines and in perspective view is shown in Figure 11.
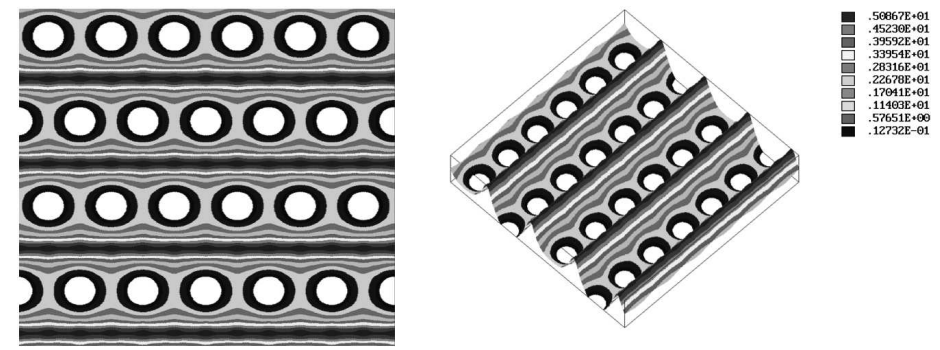

Figure 11. Plots of the function $\omega_{d_{12}, d_{14}, d_{21}}(x, y)$ for subsection 4.7.

\subsection{Perforated domain with elements of perforation grid forms the} vertices of an isosceles trapezium

Let us complicate a case of the perforated domain and consider the one when its elements form the vertices of an isosceles trapezium. For this case, the boundary equation of domain $\Omega$ is represented as

$$
\begin{aligned}
& \omega_{d_{12}, d_{13}, d_{14}, d_{21}}(x, y) \equiv-\left[\left\{\omega_{1}\left(\mu\left(x, d_{14}\right), \mu\left(y, d_{21}\right)\right) \wedge_{0} \cos \left(\mu\left(y, d_{21}\right)\right)\right\}\right. \\
& \quad \vee_{0}\left\{\left\{\omega_{1}\left(\mu\left(x-d_{12}, 2 d_{14}\right), \mu\left(y-d_{21}, d_{21}\right)\right)\right.\right. \\
& \left.\left.\left.\vee_{0} \omega_{1}\left(\mu\left(x-d_{13}, 2 d_{14}\right), \mu\left(y-d_{21}, d_{21}\right)\right)\right\} \wedge_{0} \cos \left(\mu\left(y-d_{21}, d_{21}\right)\right)\right\}\right]=0 .
\end{aligned}
$$

Set values of numeric parameters in the Equation (4.3) with the next: $12 d_{12}=$ $4 d_{13}=3 d_{14}=6 d_{21}=24 a$. For this case plots of the function $\omega_{d_{12}, d_{13}, d_{14}, d_{21}}(x, y)$ as a picture of the level lines and in perspective view is shown in Figure 12.

4.9 Perforated domain with elements of perforation grid forms the vertices of an isosceles trapezium with a longer length along the $x$-axis

Let change values of the parameters in Equation (4.3) so that the elements of the perforation grid $\Omega_{0}$ form the vertices of an isosceles trapezium with a 

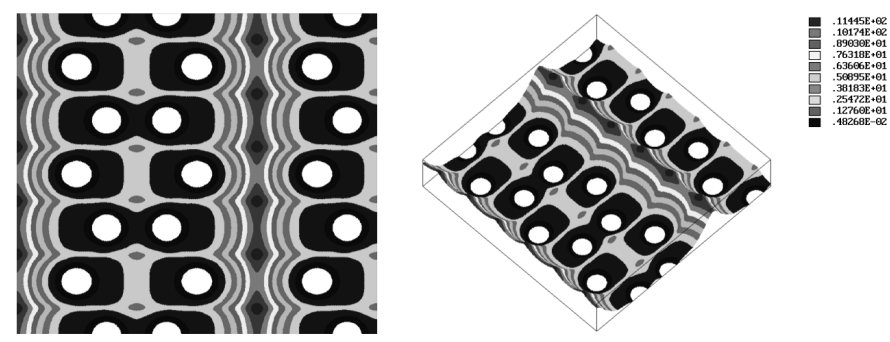

Figure 12. Plots of the function $\omega_{d_{12}, d_{13}, d_{14}, d_{21}}(x, y)$ for subsection 4.8 .

longer length along the $x$-axis, that is $20 d_{12}=5 d_{13}=4 d_{14}=10 d_{21}=40 a$. Then plots of the function $\omega_{d_{12}, d_{13}, d_{14}, d_{21}}(x, y)$ as a picture of the level lines and in perspective view is shown in Figure 13.
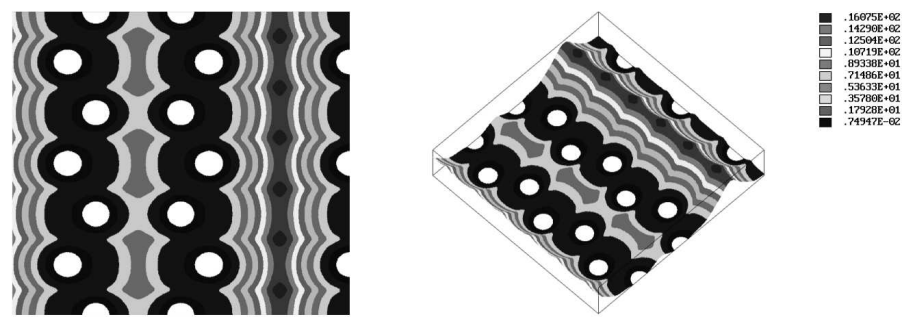

Figure 13. Plots of the function $\omega_{d_{12}, d_{13}, d_{14}, d_{21}}(x, y)$ for subsection 4.9.

4.10 Perforated domain with elements of perforation grid forms the vertices of an isosceles trapezium with a longer length along the $y$-axis

If change values of the numerical parameters to the following: $12 d_{12}=4 d_{13}=$ $3 d_{14}=4 d_{21}=24 a$, then it result to case when the elements of the perforation grid $\Omega_{0}$ form the vertices of an isosceles trapezium with a longer length along the $y$-axis. Plots of the function $\omega_{d_{12}, d_{13}, d_{14}, d_{21}}(x, y)$ as a picture of the level lines and in perspective view is shown in Figure 14.
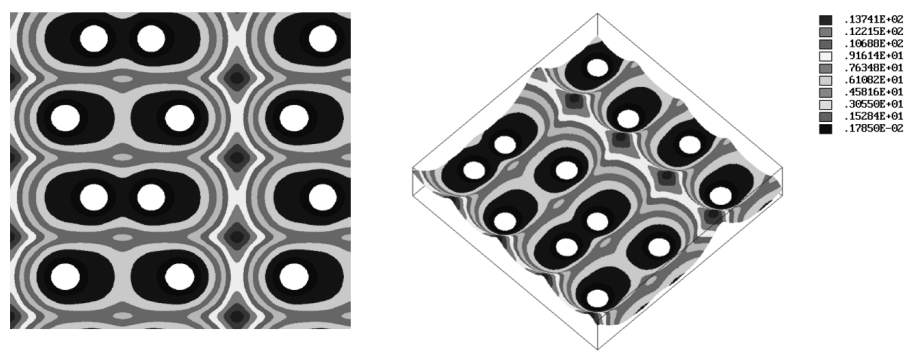

Figure 14. Plots of the function $\omega_{d_{12}, d_{13}, d_{14}, d_{21}}(x, y)$ for subsection 4.10. 
4.11 Perforated domain with elements of perforation grid forms the vertices of a rectangular trapezium

Let us consider more complicated case of a geometric figure with vertices that are elements of the perforation grid $\Omega_{0}$. Suppose it would be a rectangular trapezium. In this case, values of the numerical parameters in the Equation (4.3) were set as $d_{12}=0,3 d_{13}=2 d_{14}=3 d_{21}=12 a$. Then plots of the function $\omega_{d_{12}, d_{13}, d_{14}, d_{21}}(x, y)$ as a picture of the level lines and in perspective view is shown in Figure 15.
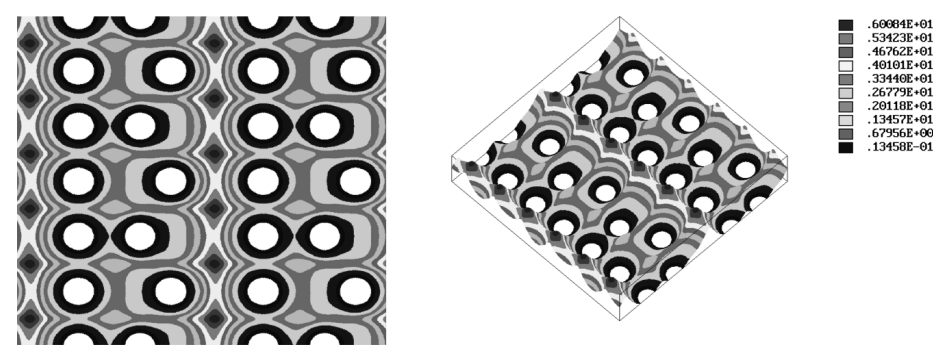

Figure 15. Plots of the function $\omega_{d_{12}, d_{13}, d_{14}, d_{21}}(x, y)$ for subsection 4.11.

4.12 Perforated domain with elements of perforation grid forms the vertices of a rectangular trapezium with a longer length along the $x$-axis

Change values of the numerical parameters in the Equation (4.3) so that elements of the perforation grid $\Omega_{0}$ form the vertices of a rectangular trapezium with a longer length along the $x$-axis, that is $d_{12}=0,4 d_{13}=3 d_{14}=6 d_{21}=24 a$. As a result, obtain plots of the function $\omega_{d_{12}, d_{13}, d_{14}, d_{21}}(x, y)$ of such domain as a picture of the level lines and in perspective view is shown in Figure 16.
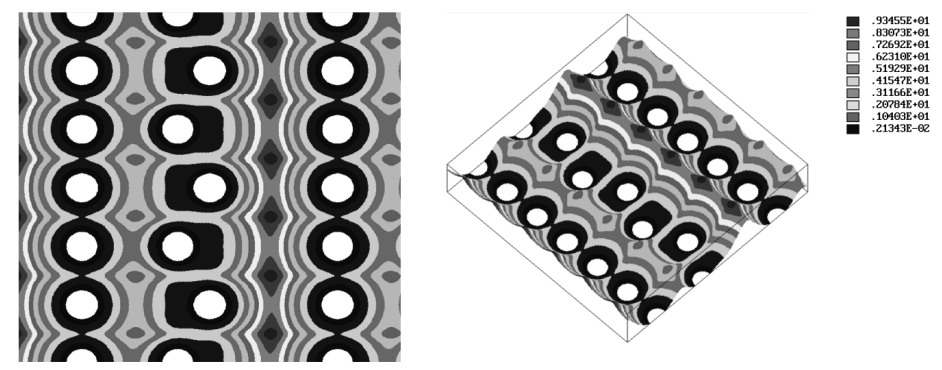

Figure 16. Plots of the function $\omega_{d_{12}, d_{13}, d_{14}, d_{21}}(x, y)$ for subsection 4.12 . 
4.13 Perforated domain with elements of perforation grid forms the vertices of a rectangular trapezium with a longer length along the $y$-axis

For a case of values the numerical parameters are set to $d_{12}=0,3 d_{13}=2 d_{14}=$ $2 d_{21}=12 a$, the elements of the perforation grid $\Omega_{0}$ form the vertices of a rectangular trapezium with a longer length along the $y$-axis. Plots of the function $\omega_{d_{12}, d_{13}, d_{14}, d_{21}}(x, y)$ as a picture of the level lines and in perspective view is shown in Figure 17.
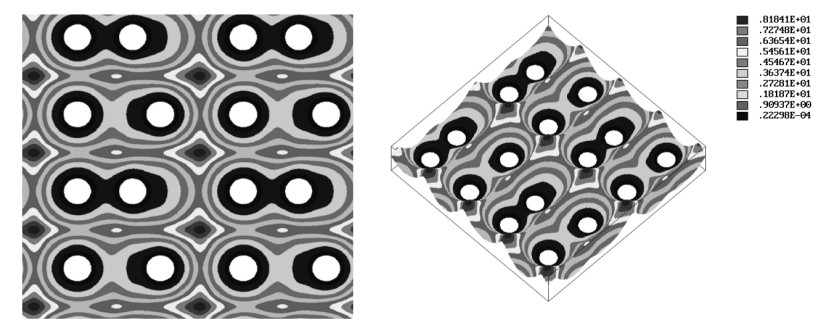

Figure 17. Plots of the function $\omega_{d_{12}, d_{13}, d_{14}, d_{21}}(x, y)$ for subsection 4.13 .

\subsection{Perforated domain with non symmetry elements of perforation grid}

It should be note that all considered equations describing by the formulas (4.1), (4.2) and (4.3) were used for cases when element "1" of the perforation grid $\Omega_{0}$ has symmetry along the coordinate axes. If this condition is not fulfilled, then the proposed method will require modification.

For example, let choose an element "1" for which the boundary equation has the form

$$
\begin{gathered}
\omega_{1}^{\prime}(x, y) \equiv\left\{\omega_{1}(x, y) \vee_{0} \omega_{1}((x-1),(y-1)) \vee_{0} \omega_{1}((x+1),(y+1))\right\} \\
\wedge_{0}\left\{\left(-\omega_{1}((x+1),(y-1))\right) \wedge_{0}\left(-\omega_{1}((x-1),(y+1))\right)\right\}=0 .
\end{gathered}
$$

Plots of the function for $\omega_{1}^{\prime}(x, y)$ is shown in Figure 18 as a picture of the level lines and in a perspective view.
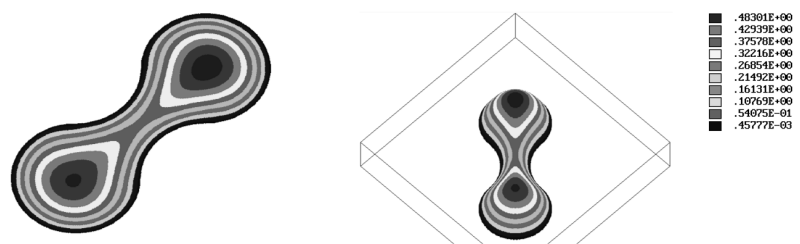

Figure 18. Plots of the function $\omega_{1}^{\prime}(x, y)$. 
Obviously, because the function $\omega_{1}^{\prime}(x, y)$ has no symmetry relative to the $x$-axis and $y$-axis, it is not even to coordinates $x$ and $y$, i.e. $\omega_{1}^{\prime}(-x,-y) \neq$ $\omega_{1}^{\prime}(x, y)$.

In this regard, the use of this function in equation (4.1) will lead to the perforated domain shown in Figure 19.
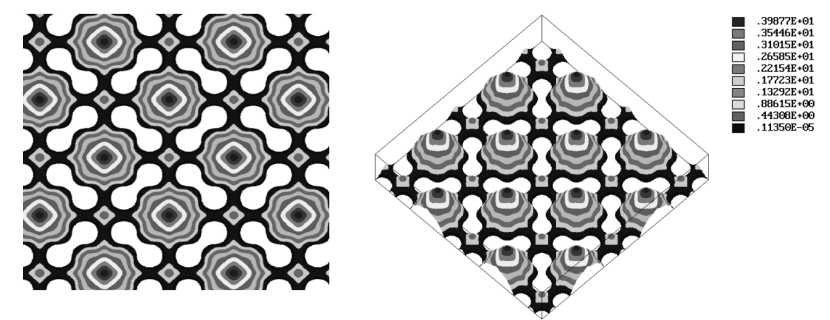

Figure 19. Plots of the function $\omega_{d_{14}, d_{21}}(x, y)$.

Here, due to properties of the functions $\mu\left(x, d_{14}\right)$ and $\mu\left(y, d_{21}\right)$ it is seen that elements of the perforation grid $\Omega_{0}$ have the symmetry relative to each other. Of course, this case of the perforated domain has some interest, but, nevertheless, it does not meet the original requirement. Therefore, we propose the following method for constructing a boundary equation of a perforated domain in this case:

$$
\begin{aligned}
& \omega_{d_{14}, d_{21}}(x, y) \equiv-\left[\left\{\omega_{1}^{\prime}\left(\mu\left(x, d_{14}\right), \mu\left(y, d_{21}\right)\right) \wedge_{0} \cos \left(\mu\left(x, d_{14}\right)\right)\right.\right. \\
& \left.\wedge_{0} \cos \left(\mu\left(y, d_{21}\right)\right)\right\} \vee_{0}\left\{\omega_{1}^{\prime}\left(\mu\left(x-d_{21}, d_{21}\right), \mu\left(y, d_{21}\right)\right) \wedge_{0} \cos \left(\mu\left(x-d_{21}, d_{21}\right)\right)\right. \\
& \left.\wedge_{0} \cos \left(\mu\left(y, d_{21}\right)\right)\right\} \vee_{0}\left\{\omega_{1}^{\prime}\left(\mu\left(x, d_{14}\right), \mu\left(y-d_{21}, d_{21}\right)\right) \wedge_{0} \cos \left(\mu\left(x, d_{14}\right)\right)\right. \\
& \left.\wedge_{0} \cos \left(\mu\left(y-d_{21}, d_{21}\right)\right)\right\} \vee_{0}\left\{\omega_{1}^{\prime}\left(\mu\left(x-d_{21}, d_{21}\right), \mu\left(y-d_{21}, d_{21}\right)\right)\right. \\
& \left.\left.\wedge_{0} \cos \left(\mu\left(x-d_{21}, d_{21}\right)\right) \wedge_{0} \cos \left(\mu\left(y-d_{21}, d_{21}\right)\right)\right\}\right]=0
\end{aligned}
$$

Values of the numerical parameters were chosen as follows: $d_{14}=d_{21}=5 a$. In this case, plots of the function $\omega_{d_{14}, d_{21}}(x, y)$ as a picture of the level lines and in perspective view is shown in Figure 20.
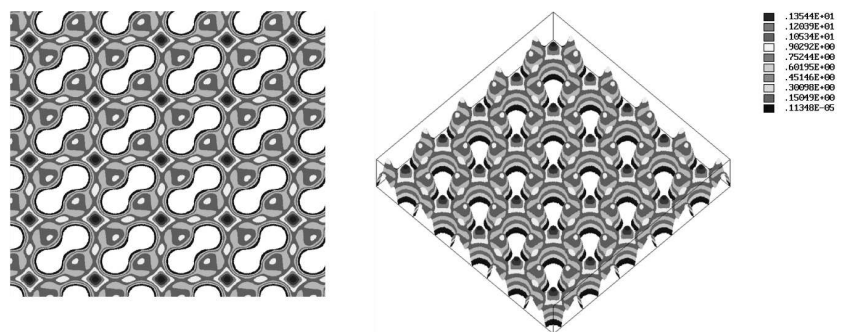

Figure 20. Plots of the function $\omega_{d_{14}, d_{21}}(x, y)$ for subsection 4.14.

It is important to note, all considered above different types of geometric figures that form elements of the perforation grid can be received by using the function $\omega_{1}^{\prime}(x, y)$ instead of the function $\omega_{1}(x, y)$. 


\subsection{Perforated domain with non separated elements of perforation grid}

Therefore, we now turn to the consideration of the last case, which differs from all previous ones in that the elements of the perforation grid $\Omega_{0}$ cannot be separated from each other by means of strips along the $x$-axis and the $y$-axis.

For example, let choose an element "1" for which the boundary equation has the form

$$
\begin{gathered}
\omega_{1}^{\prime \prime}(x, y) \equiv\left(\omega_{11}(-x+5, y) \wedge_{0} \omega_{11}(x+1,-y)\right) \wedge_{0}\left(\omega_{11}(x+5, y)\right. \\
\left.\wedge_{0} \omega_{11}(-x+5,-y)\right) \vee_{0}\left(\omega_{11}(-x+1, y) \wedge_{0} \omega_{11}(x+5,-y)\right) \\
\wedge_{0}\left(\omega_{11}(x+1, y) \wedge_{0} \omega_{11}(-x+1,-y)\right)=0
\end{gathered}
$$

where $\omega_{11}(x, y)=y+x$.

Plots of the function $\omega_{1}^{\prime \prime}(x, y)$ is shown in Figure 21 as a picture of the level lines and in a perspective view.
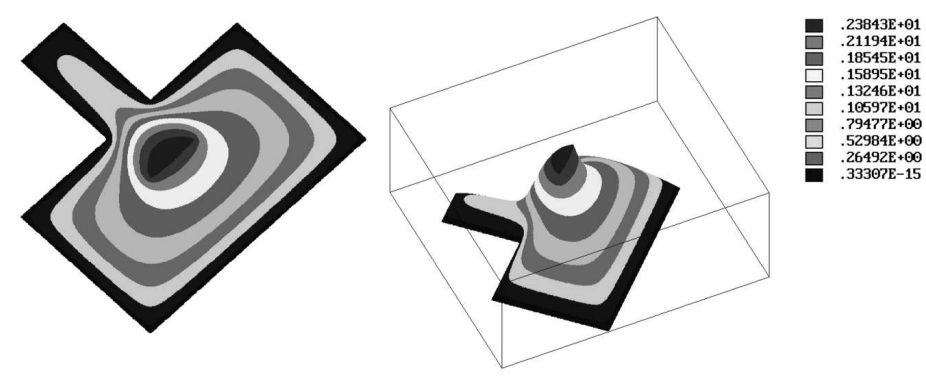

Figure 21. Plots of the function $\omega_{1}^{\prime \prime}(x, y)$.

The boundary equation of the perforated domain, for which the functiondefined domain $\omega_{1}^{\prime \prime}(x, y)$ is used as the perforation grid element, has the form

$$
\begin{aligned}
& \omega_{d_{14}^{\prime}, d_{21}^{\prime}}(x, y) \equiv-\left[\left\{\omega_{1}^{\prime \prime}\left(\mu\left(x, d_{14}^{\prime}\right), \mu\left(y, d_{21}^{\prime}\right)\right) \wedge_{0} \cos \left(\mu\left(x, d_{14}^{\prime}\right)\right) \wedge_{0} \cos \left(\mu\left(y, d_{21}^{\prime}\right)\right)\right\}\right. \\
& \vee_{0}\left\{\omega_{1}^{\prime \prime}\left(\mu\left(x-d_{21}^{\prime}, d_{21}^{\prime}\right), \mu\left(y, d_{21}^{\prime}\right)\right) \wedge_{0} \cos \left(\mu\left(x-d_{21}^{\prime}, d_{21}^{\prime}\right)\right) \wedge_{0} \cos \left(\mu\left(y, d_{21}^{\prime}\right)\right)\right\} \\
& \vee_{0}\left\{\omega_{1}^{\prime \prime}\left(\mu\left(x, d_{14}^{\prime}\right), \mu\left(y-d_{21}^{\prime}, d_{21}^{\prime}\right)\right) \wedge_{0} \cos \left(\mu\left(x, d_{14}^{\prime}\right)\right) \wedge_{0} \cos \left(\mu\left(y-d_{21}^{\prime}, d_{21}^{\prime}\right)\right)\right\} \\
& \vee_{0}\left\{\omega_{1}^{\prime \prime}\left(\mu\left(x-d_{21}^{\prime}, d_{21}^{\prime}\right), \mu\left(y-d_{21}^{\prime}, d_{21}^{\prime}\right)\right) \wedge_{0} \cos \left(\mu\left(x-d_{21}^{\prime}, d_{21}^{\prime}\right)\right)\right. \\
& \left.\wedge_{0} \cos \left(\mu\left(y-d_{21}^{\prime}, d_{21}^{\prime}\right)\right)\right\} \vee_{0}\left\{\omega_{1}^{\prime \prime}\left(\mu\left(x-d_{21}, d_{21}^{\prime}\right), \mu\left(y, d_{21}^{\prime}\right)\right) \wedge_{0} \cos \left(\mu\left(x-d_{21}, d_{21}^{\prime}\right)\right)\right. \\
& \left.\wedge_{0} \cos \left(\mu\left(y, d_{21}^{\prime}\right)\right)\right\} \vee_{0}\left\{\omega_{1}^{\prime \prime}\left(\mu\left(x-3 d_{21}, d_{21}^{\prime}\right), \mu\left(y, d_{21}^{\prime}\right)\right) \wedge_{0} \cos \left(\mu\left(x-3 d_{21}, d_{21}^{\prime}\right)\right)\right. \\
& \left.\wedge_{0} \cos \left(\mu\left(y, d_{21}^{\prime}\right)\right)\right\} \vee_{0}\left\{\omega_{1}^{\prime \prime}\left(\mu\left(x-d_{21}, d_{21}^{\prime}\right), \mu\left(y-d_{21}^{\prime}, d_{21}^{\prime}\right)\right) \wedge_{0} \cos \left(\mu\left(x-d_{21}, d_{21}^{\prime}\right)\right)\right. \\
& \left.\wedge_{0} \cos \left(\mu\left(y-d_{21}^{\prime}, d_{21}^{\prime}\right)\right)\right\} \vee_{0}\left\{\omega_{1}^{\prime \prime}\left(\mu\left(x-3 d_{21}, d_{21}^{\prime}\right), \mu\left(y-d_{21}^{\prime}, d_{21}^{\prime}\right)\right)\right. \\
& \left.\wedge_{0} \cos \left(\mu\left(x-3 d_{21}, d_{21}^{\prime}\right)\right) \wedge_{0} \cos \left(\mu\left(y-d_{21}^{\prime}, d_{21}^{\prime}\right)\right)\right\} \vee_{0}\left\{\omega _ { 1 } ^ { \prime \prime } \left(\mu\left(x, d_{14}^{\prime}\right),\right.\right. \\
& \left.\left.\mu\left(y-d_{21}, d_{21}^{\prime}\right)\right) \wedge_{0} \cos \left(\mu\left(x, d_{14}^{\prime}\right)\right) \wedge_{0} \cos \left(\mu\left(y-d_{21}, d_{21}^{\prime}\right)\right)\right\} \\
& \vee_{0}\left\{\omega_{1}^{\prime \prime}\left(\mu\left(x-d_{21}^{\prime}, d_{21}^{\prime}\right), \mu\left(y-d_{21}, d_{21}^{\prime}\right)\right) \wedge_{0} \cos \left(\mu\left(x-d_{21}^{\prime}, d_{21}^{\prime}\right)\right)\right. \\
& \left.\left.\wedge_{0} \cos \mu\left(y-d_{21}, d_{21}^{\prime}\right)\right)\right\} \vee_{0}\left\{\omega_{1}^{\prime \prime}\left(\mu\left(x, d_{14}^{\prime}\right), \mu\left(y-3 d_{21}, d_{21}^{\prime}\right)\right) \wedge_{0} \cos \left(\mu\left(x, d_{14}^{\prime}\right)\right)\right.
\end{aligned}
$$




$$
\begin{gathered}
\left.\wedge_{0} \cos \left(\mu\left(y-3 d_{21}, d_{21}^{\prime}\right)\right)\right\} \vee_{0}\left\{\omega_{1}^{\prime \prime}\left(\mu\left(x-d_{21}^{\prime}, d_{21}^{\prime}\right), \mu\left(y-3 d_{21}, d_{21}^{\prime}\right)\right)\right. \\
\left.\wedge_{0} \cos \left(\mu\left(x-d_{21}^{\prime}, d_{21}^{\prime}\right)\right) \wedge_{0} \cos \left(\mu\left(y-3 d_{21}, d_{21}^{\prime}\right)\right)\right\} \\
\vee_{0}\left\{\omega_{1}^{\prime \prime}\left(\mu\left(x-d_{21}, d_{21}^{\prime}\right), \mu\left(y-d_{21}, d_{21}^{\prime}\right)\right) \wedge_{0} \cos \left(\mu\left(x-d_{21}, d_{21}^{\prime}\right)\right)\right. \\
\left.\wedge_{0} \cos \left(\mu\left(y-d_{21}, d_{21}^{\prime}\right)\right)\right\} \vee_{0}\left\{\omega_{1}^{\prime \prime}\left(\mu\left(x-3 d_{21}, d_{21}^{\prime}\right), \mu\left(y-d_{21}, d_{21}^{\prime}\right)\right)\right. \\
\left.\wedge_{0} \cos \left(\mu\left(x-3 d_{21}, d_{21}^{\prime}\right)\right) \wedge_{0} \cos \left(\mu\left(y-d_{21}, d_{21}^{\prime}\right)\right)\right\} \\
\vee_{0}\left\{\omega_{1}^{\prime \prime}\left(\mu\left(x-d_{21}, d_{21}^{\prime}\right), \mu\left(y-3 d_{21}, d_{21}^{\prime}\right)\right)\right. \\
\left.\wedge_{0} \cos \left(\mu\left(x-d_{21}, d_{21}^{\prime}\right)\right) \wedge_{0} \cos \left(\mu\left(y-3 d_{21}, d_{21}^{\prime}\right)\right)\right\} \\
\vee_{0}\left\{\omega_{1}^{\prime \prime}\left(\mu\left(x-3 d_{21}, d_{21}^{\prime}\right), \mu\left(y-3 d_{21}, d_{21}^{\prime}\right)\right)\right. \\
\left.\left.\wedge_{0} \cos \left(\mu\left(x-3 d_{21}, d_{21}^{\prime}\right)\right) \wedge_{0} \cos \left(\mu\left(y-3 d_{21}, d_{21}^{\prime}\right)\right)\right\}\right]=0 .
\end{gathered}
$$

Plots of the function $\omega_{d_{14}^{\prime}, d_{21}^{\prime}}(x, y)$ shown in Figure 22 for $d_{14}=d_{21}=7 a$.
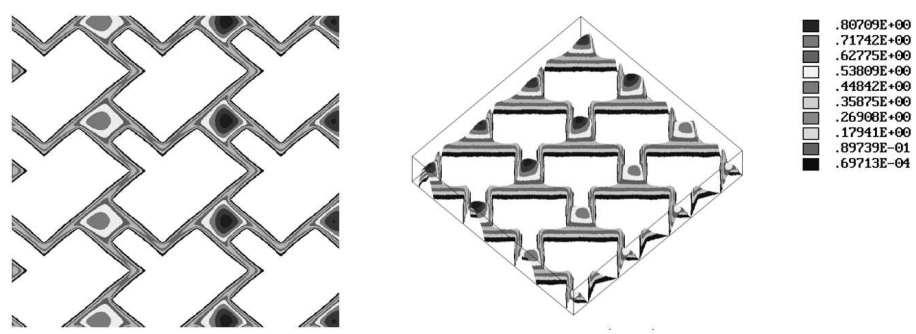

Figure 22. Plots of the function $\omega_{d_{14}^{\prime}, d_{21}^{\prime}}(x, y)$ for subsection 4.15 .

Here, for convenience, the notation $d_{14}^{\prime}=2 d_{14}, d_{21}^{\prime}=2 d_{21}$ is used.

\section{Conclusions}

Thus, in this paper the problem of constructing the boundary equations for perforated geometric domains is considered for the first time. This is undoubtedly the novelty of the presented research and contribution in the R-functions theory development. The proposed equations can be used to solve boundary problems of mathematical physics in perforated domains by the help of the R-functions method. All results presented in the work were visualized by the Polye system developed by a team of scientists from the Institute for Problems of Mechanical Engineering of the National Academy of Sciences of Ukraine (Kharkov) under the guidance of Academician V.L. Rvachev (21.10.1926-26.04.2005). The results presented in this paper were obtained for the first time and have not been published before.

\section{Acknowledgements}

I would like to show my gratitude to Mr. Riivo Must for interest in this paper. During a discussion of the results, very significant recommendations were done by him that would improve the final appearance of the paper. Also, I thank the organizing committee of the International Conference Mathematical Modelling and Analysis 2019 for the opportunity to make a presentation of received results at the conference in Tallinn. 


\section{References}

[1] F. Bauer and E.L. Reiss. Stresses in a perforated cylindrical shell. International Journal of Solids and Structures, 2(2):141-156, 1966.

[2] E.I. Grigolyuk and L.A. Filshtinsky. Perforated plates and shells. Nauka, Moscow (in Russian), 1970.

[3] C. Kingery, R. Pearson and G. Coulter. Shock wave attenuation by perforated plates with various hole sizes. In USA Ballistic Research Laboratory(Ed.), BRL Memorandum report No 275\%, p. 71, Maryland 21005, 1977. Aberdeen Proving Ground.

[4] V.L. Rvachev. Theory of R-functions and some applications. Naukova Dumka, Kiev (in Russian), 1982.

[5] V.L. Rvachev and T.I. Sheiko. R-functions in boundary value problems in mechanics. Applied Mechanics Reviews, 48(4):151-188, 1995. https://doi.org/10.1115/1.3005099.

[6] Yu.S. Semerich. The R-functions method in boundary value problem for complex domain possessing symmetry. In Abstracts of the Seventh International Conference Mathematical Modelling and Analysis MMA2002, p. 55. Kaariku, Estonia, May 31-June 2, 2002, 2002.

[7] Yu.S. Semerich. The R-functions method in the boundary value problem for a complex domain possessing the symmetry. Mathematical modelling and analysis, 8(1):77-86, 2003. https://doi.org/10.3846/13926292.2003.9637212.

[8] Yu.S. Semerich. The construction of loci with a cyclical symmetry by the R-functions. Mathematical modelling and analysis, 10(1):73-82, 2005. https://doi.org/10.3846/13926292.2005.9637272.

[9] H. Weyl. Symmetry. Princeton University Press, Princeton, New Jersey, 1952. 